\section{Preferred recall order and recall accuracy for two messages presented simultaneously
over a single auditory channel*}

HOWARD ROLLINS, MARK EVERSON, and DONALD L. SCHURMAN† Emory University, Atlanta, Georgia 30322

Ss in the dichotic listening task prefer, and are more accurate with, the channel-by-channel order of recall-recalling all information presented to one ear followed by the information presented to the other ear. Current explanations for these effects rely upon the fact that the two messages are presented over separate in put channels. The present study tested this hypothesis directly by presenting two messages simultaneously to a single auditory channel. Groups of Ss were instructed to recall two pairs of digits presented simultaneously over a single channel (1) in any order (free recall), (2) in the order received (simultaneous order), or (3) in sequential groups (successive order). Ss in the free recall task preferred recalling items in successive order. However, Ss instructed to recall in simultaneous order were as accurate as those instructed to recall in successive order. These data imply that accuracy with, but not preference for, the successive order of recall depends upon whether input is to one or two channels.

Ss in the dichotic listening task prefer to recall all the information presented to one ear followed by the information presented to the other ear-in a channel-by-channel order (Broadbent, 1954; Bryden, 1962). Further, Ss are more accurate when instructed to recall by channel than by simultaneous pair (Broadbent, 1954; Moray, 1960). Broadbent (1957) and Yntema and Trask (1963) assume, without supporting data, that these effects are prodcued by presenting the two messages over separate channels.

Savin (1967) tested this assumption in a free recall paradigm. His Ss received two pairs of simultaneous auditory digits over a single channel. Each digit of a pair was recorded on a channel of a stereo tape, then the two channels played back over a single speaker. The majority of Savin's Ss preferred recalling the items in successive order, recalling one item of the first pair, then an item of the next pair, followed by the remaining item of each pair, rather than recalling the items in the order received (i.e., in simultaneous pairs). Since the successive order of recall is analogous to channel order of recall when input is to separate channels, Savin concluded that successive processing has nothing to do with the number of channels over which auditory information is presented.

Since Savin (1967) used the free

*This research was supported by Grant No. 4182 from Emory University to the first author.

tRequests for reprints should be sent to Howard Rollins, Department of Psychology Emory University, Atlanta, Georgia 30322. recall paradigm and Ss' preferences as his dependent variable, his conclusions may apply only to the preferred order of recall and not to recall accuracy. In the experiment reported here, groups of $\mathrm{Ss}$ were instructed to recall two messages presented over a single channel (1) in any order, (2) in successive pairs, or (3) in simultaneous pairs. The first group replicated Savin's experiment. The latter two groups two orders of recall.

\section{METHOD}

Stimulus Materials

Each trial consisted of two pairs of simultaneous digits sampled randomly without replacement from the set 1-9. The second pair began .5 sec after the beginning of the first pair. One digit of each simultaneous pair was recorded on each track of a stereo tape recorder (Sony Model TC-540), following Savin (1967). Both tracks were played back through a single speaker.

In order to ascertain the simultaneity of and the intensity levels of each simultaneous pair, the recordings of each trial were examined with a Tektronix oscilloscope (Model No. Rm5031). The mean absolute deviation from simultaneity for the 80 stimulus pairs was 32.75 msec. The peak intensity for each of the four digits on a single trial was at most $38.0 \mathrm{~dB}$ and at least $26.0 \mathrm{~dB}(0.1-\mathrm{V}$ reference level).

\section{Procedure}

All Ss were run individually. Ss in the free recall group were instructed to repeat all four digits, in the order that they found easiest, as soon as the last assessed the relative difficulty of the digit had been presented. Ss in the simultaneous recall group were instructed to repeat both digits of the first pair followed by both digits of the second pair. Ss in the successive recall group were told to repeat a digit from the first pair, then a digit from the second pair, followed by the remaining digit from the first pair and finally by the remaining digit from the second pair. Ss in the latter two groups were shown a visual illustration of a "typical" trial.

Each $S$ received each of six practice trials until he was able to recall all four digits correctly and, if in one of the instructed recall groups, until he repeated them in the correct order. Ss unable to recall any practice trial correctly in nine attempts were dropped from the study. Thirteen Ss were excluded in this way (free recall, four Ss; simultaneous recall, seven Ss; successive recall, two $\mathrm{Ss}$ ).

Ss received 40 experimental trials without feedback. If a $S$ in one of the instructed recall groups had difficulty ordering the digits correctly (i.e., incorrectly ordered three or more trials in a row), the $E$ reexplained the ordering instructions. Intertrial time for each trial was approximately 6-8 sec.

Ss in the free recall group were then dismissed. Ss in the two instructed recall groups recalled an additional 10 trials in a different order. Simultaneous recall Ss were instructed to recall in successive order, successive recall Ss in simultaneous order. Verbal and visual explanations of the correct orders of recall were given as before. Again, Ss were given no practice or feedback.

\section{Subjects}

Twelve graduate and undergraduate students attending Emory University were assigned randomly to each of the three experimental groups. An equal number of males and females were assigned to each group. All Ss were paid for participation.

\section{RESULTS}

The data were scored in two ways: (1) the percentage of trials recalled without error and (2) the percentage of trials recalled correctly and in simultaneous, successive, or some other order (mixed). These dependent variables are presented in Table 1 for the three groups.

It is evident in Table 1 that $S s$ in the free recall group displayed a strong preference for successive orders of recall. These Ss recalled approximately twice as many trials correctly in successive orders as in simultaneous orders. Seven of the 12 Ss recalled over $75 \%$ of their correct trials in successive orders; only two Ss showed 
Table 1

Mean Percentage of Total Trials Recalled Without Error and in Successive, Simultaneous, or Some Other Order (Mixed) as a Function of Recall Condition

\begin{tabular}{lcccc}
\hline & \multicolumn{2}{c}{ Order of Recall } \\
\cline { 2 - 5 } Recall Condition & $\begin{array}{c}\text { Succes- } \\
\text { ive Mean } \\
\text { Percentage }\end{array}$ & $\begin{array}{c}\text { Simultan- } \\
\text { eous Mean } \\
\text { Percentage }\end{array}$ & $\begin{array}{c}\text { Mixed } \\
\text { Mean } \\
\text { Percentage }\end{array}$ & $\begin{array}{c}\text { Total } \\
\text { Mean } \\
\text { Percentage }\end{array}$ \\
\hline $\begin{array}{l}\text { Free Recall } \\
\text { Instructed } \\
\text { Successive Order }\end{array}$ & 49 & 24 & 6 & 79 \\
$\begin{array}{c}\text { Instructed } \\
\text { Simultaneous Order }\end{array}$ & 76 & 6 & 4 & 86 \\
\hline
\end{tabular}

a clear preference for simultaneous orders of recall $(83 \%$ and $90 \%$ simultaneous order, respectively). The rank order correlation between number of trials recalled correctly and the ratio of the number of trials recalled in successive to simultaneous orders was $0.54(p<.05)$. Thus, Ss preferring successive orders of recall were also more accurate. These results are consistent with the data reported by Savin (1967).

As indicated in Column 4 of Table 1, Ss in the successive recall group recalled a higher percentage of trials without error than did Ss in either the free recall group or the simultaneous recall group. An analysis of variance comparing these three means was not significant, $F(2,33)=2.35, p>.05$.

Columns 2 and 3 of Table 1 provide a direct comparison between the two instructed orders of recall. Ss in the successive recall group recalled $76 \%$ of the trials correctly and in successive order, whereas $S s$ in the simultaneous recall group recalled only $68 \%$ of the trials correctly and in simultaneous order. This $8 \%$ difference in recall accuracy did not approach significance, $F(1,22)=1.32, p>.05$.

Many of the Ss in the present experiment reported that one digit of a simultaneous pair appeared louder or in a different frequency from the other. These reports suggest that for some trials Ss were receiving the two messages over two channels. In order to examine this possibility, all trials for the successive recall group were inspected for evidence of some form of two-channel input. Ss in this group recalled the information in one of two successive orders: either with the stereo channels (recalling together the digit of each pair recorded on one track of the tape) or across them. If the information is arriving over a single channel, then Ss should be as likely to recall in either of these successive orders. For each trial, a frequency count was made of the number of Ss recalling the digits in successive order and either with the stereo channels or across them. The ratio of the larger of these numbers over the total number of Ss recalling the digits without gross errors was used as an index of the presence of biased trials. A number of trials were biased by this index. The 10 trials with the lowest bias index had a mean index ratio of .56 ; the 10 trials with the highest bias index had a mean index ratio of .80 .

The major focus of the present study is the efficiency of the two orders of recall when two messages are presented over a single channel. Clearly, the 10 trials with the lowest bias index fit this criterion. However, for those trials with a large bias index, some perceived difference between input items, i.e., some sort of perceptual channeling, may occur systematically. These two types of trials may differentially affect the accuracy for the two orders of recall. Consequently, the 10 high bias trials and the 10 low bias trials were included as a repeated measure factor in a 2 by 2 analysis of variance with instructed order of recall as the second factor. The dependent variable was percentage of trials recalled correctly and in the instructed order. Degree of bias interacted with instructed order of recall, $F(1,22)=4.44, p<.05$. For the low-bias trials, Ss performed equally well with either the successive or the simultaneous order of recall, mean percent correct $=70$ and 72 , respectively. For the high-bias trials, Ss recalling in successive order were reliably more accurate than Ss recalling in simultaneous order, mean percent correct $=85$ and 72 , respectively. Degree of bias did not affect preference for the two orders of recall since on $73 \%$ of the unbiased and $68 \%$ of the biased trials, Ss in the free recall group preferred to recall in successive order, $t(11)=1.00, p>.05$.

Accuracy of recall improved across the four 10-trial blocks (mean percent correct $=68 \%, 66 \%, 72 \%, 80 \%$, respectively), $F(3,66)=4.18, p<.05$. For the fifth block of trials, Ss were asked to recall in successive order if the first 40 trials were simultaneous and vice versa. This procedure resulted in a significant decrease (by
Newman-Keuls procedure) in the number of correct trials in Block 5 ( mean percent correct $=69 \%$ ) to the level of the first 10-trial block. The effect of trials was not a function of order since trials and order did not interact, $F(3,66)=1.53, p>.05$.

The mean number of trials required to reach the practice-trial criterion was identical for both instructed recall groups (mean $=13.33$ ).

\section{DISCUSSION}

Given free choice, most Ss recall simultaneous auditory information in successive order. Apparently, this phenomenon occurs whether the two messages arrive over a single channel, as in Savin's (1967) experiment and in the present study, or two channels, as in previous research by Broadbent (1954) and Bryden (1962). Savin suggests that this result is a function of everyday experiences with the processing of auditory information. It is usually more useful to follow and attend to a single auditory message than to collate two auditory messages. After all, verbal material is primarily sequential in nature. Most of the simultaneously occurring dimensions of verbal stimuli-intensity, harmonics, frequency range-can be changed or greatly diminished without destroying the inteligibility of the material.

On the other hand, Madsen, Rollins, and Senf (1970) have demonstrated that S's preferences in an analogous bisensory task are easily influenced both by the instructions they receive and by the experience of the first few trials. The order initially chosen was usually continued, in spite of changes in the task that required a different recall order for efficient performance. It is possible, therefore, that either the instructions used or the initial practice trials led most of our Ss (and Savin's) to view the stimuli as successive sets rather than simultaneous pairs.

The accuracy data of the present experiment indicate that two messages presented simultaneously over a single channel are recalled as accurately in the order received (i.e., simultaneously) as successively. This result is in contrast to the consistent finding of superior accuracy with successive recall when input is to two channels (Broadbent, 1954; Broadbent \& Gregory, 1961; Yntema \& Trask, 1963). It would appear, then, that even though Ss prefer successive orders of recall with input to a single channel this order leads to greater accuracy only when some form of two-channel input is present. This conclusion is consistent with the theoretical interpretations of both Broadbent (1957) and Yntema and Trask (1963).

While it is clear that both Broadbent (1957) and Yntema and Trask (1963) 
predict superior recall in successive order when input is to two channels, it is not apparent what predictions are made concerning the presentation of two messages over a single channel. One may predict either that simultaneous order will result in more accurate recall or that there will be no difference in accuracy between the two instructed orders of recall. Broadbent (1957) suggests that information input to a single channel is processed as it arrives and is stored and retrieved in the same order. Thus, Broadbent appears to predict greater accuracy with the simultaneous order of recall. In fact, Ss are more accurate with the simultaneous order of recall when simultaneous pairs are presented slowly (e.g., one pair $/ 2$ sec). The 10 trials of the present experiment that fit most closely the criterion of single-channel input were not recalled more accurately in the simultaneous order, so these results are damaging to Broadbent's position.

Yntema and Trask (1963) propose that all information is processed and stored in nonsensory immediate memory as it arrives. As items are stored, they are given "tags" which identify, among other things, their source and time of arrival. These "tags" make possible the selective retrieval of items from immediate memory. Since pairs of items are input simultaneously in the dichotic task, Yntema and Trask (1963) argue that the order-of-arrival "tags" are not distinct and that channel (input ear) is a more useful "tag" for selective retrieval from memory. Thus, successive orders of recall result in greater accuracy. For the biased trials of the present study, it would appear that some "tag" other than order of arrival was also available to the Ss. However, for the unbiased trials, only order-of-arrival "tags" were available. If both items of the first simultaneous pair were tagged as arriving first and the two items of the second pair tagged "second," then $S$ could recall one item of the first pair followed by an item of the second pair. However, when $S$ returned to recall the remaining item of the first pair, he would be unable to determine which of the items to recall unless he could check his output list and select for the remaining first item, the one not on the output list. An inspection of the successively recalled trials in the free recall condition indicated that $S s$ confused items in the first pair on only 5 of some 323 trials. Thus, if Ss are following this strategy, they make very accurate comparisons of items on the output list and items in memory. Alternatively, the digits in the first simultaneous pair may be tagged as arriving first and also arbitrarily tagged in some other way to keep them distinct. For example, they could be compared and tagged as the numerically larger or smaller item. Either of these modifications of Yntema and Trask's original theory permit prediction of the present results.

According to Savin (1967), preference for successive orders of recall builds up through experience. If Savin is correct, then any difference between instructed successive and instructed simultaneous recall should be reduced by practice with the simultaneous order. However, practice facilitated recall in either order. These practice effects were not simply a function of general experience with the stimuli and the task. Rather, they were specific to the particular order rehearsed, since transfer to recall in the order not experienced for the first 40 trials was negative. This result suggests that Ss acquire, over the course of the experiment, some specific skills either for storing or retrieving the information in the instructed order. One possibility is that Ss develop strategies for supplying a distinct tag to each item as it is stored, such that the tagged information is more easily retrieved in the specified order.

An unusual aspect of the present study is the nature of the apparent channeling effect obtained on some trials. We anticipated that this bias would coincide with the channels of the stereo tape system. This was clearly not the case since 8 of the 18 (44\%) most biased trials were consistently recalled across the stereo channels (this proportion did not differ from chance expectation, $x^{2}(1)=0.22, p<.05$. Many of our Ss reported that they heard two sets of digits either spoken by different voices or with one digit spoken louder than the other. A comparison of simultaneity and stimulus intensity (measured on an oscilloscope) with the direction of successive recall (either with the stereo channels or across them) did not reveal any evidence that either of these variables affected recall order. Since the apparent differences of intensity or tone do not appear to be correlated with the physical characteristics of the recorded digits, we suggest that some form of masking or dominance effect may occur when certain digit pairs are presented simultaneously, with one of the two digits dominating. Therefore, one of the digits may sound more intense than or different from the other. However, our data are not sufficient to allow for any systematic analysis of the parameters of this dominance effect.

\section{REFERENCES}

BROADBENT, D. E. The role of auditory localization in attention and memory span. Journal of Experimental Psychology, 1954, 47, 191-196.

BROADBENT, D. E. A mechanical model for human attention and immediate memory. Psychological Review, 1957, 64, 205-215.

BROADBENT, D. E. Perception and communication. New York: Pergamon Press, 1958.

BROADBENT, D. E., \& GREGORY, M. On the recall of stimuli presented alternately to two sense-organs. Quarterly Joumal of Experimental Psychology, 1961, 13, 103-109.

BRYDEN, M. P. Order of report in dichotic listening. Canadian Journal of Psychology, 1962, 16, 291-299.

MADSEN, M. C.. ROLLINS, H. A., \& SENF, G. Variables affecting immediate memory for bisensory stimuli: Eye-ear analogue studies of dichotic listening. Journal of Experimental Psychology, 1970, 83, 3 (Part 2).

MORAY, N. Broadbent's filter theory: Postulate $H$ and the problem of switching time. Quarterly Journal of Experimental Psychology, 1960, 12, 214-220.

SAVIN, H. B. On the successive perception of simultaneous stimuli. Perception \& Psychophysics, 1967, 2, 479-482.

YNTEMA, D. B., \& TRASK, F. P. Recall as a search process. Journal of Verbal Learning \& Verbal Behavior, 1963, 2, 65-74

(Accepted for publication August 5, 1971.) 